\title{
A Novel Design Approach for Facial Expression of Home Robot based on Kansei Engineering
}

\author{
Yunyun Wei ${ }^{1, a^{*}}$ and Huanzhi Lou ${ }^{1, b}$ \\ ${ }^{1}$ School of Architecture and Design, Beijing Jiaotong University, Beijing, China \\ ayunyunwei@outlook.com, b14121726@bjtu.edu.cn
}

Keywords: Home robot; Facial expression; Kansei Engineering; Pearson Correlation Coefficient

\begin{abstract}
With the rapid development of robot technology, home robot is becoming more and more popular in daily life. Facial expression of robots is important to improve the interaction between human beings and robots. Kansei Engineering is used to explore users' feelings with the design of facial expression of home robot. Pearson Correlation Coefficient is one of the most popular similarity measures to evaluate the elements of home robot's face. In this paper we proposed a novel approach to evaluate the design of face and facial expression of home robot and help the designer improving user experience of the product.
\end{abstract}

\section{Introduction}

Robots are becoming more and more general machines or devices in our daily life, while the development of robot technology has been achieved in decade. Robots can be widely used regularly not only in the factory and workshop but also banks, hospitals, schools, and even more individual houses. Robots are being developed increasingly for real daily life applications, such as housekeeping, company, eldercare, and even as household assistants [1].

It is important that human beings need to feel comfortable and at ease during interaction and communication with robots. So lots of researches focus on improving the interaction between human beings and robots and achieve remarkable progresses. The improvement of interaction between human beings and robots can make people feel more comfortable and relaxing. Human-robot interaction is always the important issue for researchers and engineers. More and more studies conclude that emotions influence the interaction efficiency of human beings and robots obviously, so the recognition and expression of emotions is a key factor in the design and manufacture of robots [2].

The success of product design depends on satisfying the requirements of users in the best possible way. The most important factor in the success of home robot is to create an enjoyable experience in using the robot's functions and facing the robot's face and to meet the needs of users correctly. In order to meet user requirements and promote user experience, many methods have been developed and applied in product design based on user mental model [3]. Kansei Engineering is one of the approaches and used wildly, which was founded by Mitsuo Nagamachi [4] in 1970. It is a classical method for transforming the users' feelings and emotional effects into production parameters.

Although robot technology has achieved significant progresses in major field, there is almost no research on the form design of face and facial expression for home robot. The purpose of this paper is to propose a method which could quantify users' perceptual cognition on facial expression of home robot, present a new calculation and evaluation approach of face design of home robot based on Kansei Engineering. This paper is organized as following: In the second section, it gives an introduction of Kansei Engineering, an outline of facial expression of home robot. We propose a novel approach based on Kansei Engineering to evaluate the design of facial expression of home robot in the third section. In the fourth section, Pearson Correlation Coefficient is introduced to calculate the significance of the elements of robot face in our proposed approach. Finally, we present some conclusions and future work. 


\section{Facial Expression Design based on Kansei Engineering}

Kansei Engineering. Kansei Engineering is a classical and modern technology focused on users' feelings, which was defined as "Conveying the feelings and imagination of the customer or consumer toward a product and design elements." The designers acquire users' final feelings to analyze as input which is processed through Kansei Engineering, in order that the psychological feeling relationship with the product can be established based on all the collected information. It enables the designers to evaluate their design before launching the product into the market so that they can implement the feelings and imaginations of their users in the new product. User experience is mainly based on the emotional functionality of product. Therefore, Kansei Engineering is aimed at seeking emotional structures which were behind human behaviors or modernizing the emotional needs of users and using them in product design [5].

In Kansei designing, user requirement and experience is considered, and a relationship is established among the functional features of the product. Empirical features include the feeling which the product causes to the user. The feelings include user's attitude and emotion to the product, the way it is used and so on. In general, there are two major approaches to solve the problem based on Kansei Engineering. One is applied to evaluate and process the components of product, whereas the other one studies the product as a whole set. In Kansei Engineering, it is important that the quantitative information is derived from the qualitative information which is achieved through observation and interview. Firstly, the researchers should identify the users' requirements and feelings. After identifying users' basic evaluations to the product, the information are considered as visual signs in designing the product. Kansei words are used to express the feelings of users and can reflect Kansei elements. It is a key process of Kansei Engineering that users convey their feelings and what is on their minds with the help of the words.

In this paper, a novel approach for the design of home robot's face and facial expression is proposed based on Kansei Engineering.

Analysis of Facial Expression. According to the emotion theorists and psychologists, different emotions can be categorized starting from globally showed six fundamental emotions to complicated emotions. From several traditional researches in the field of emotion recognition, two classical models are widely used: Ekman's fundamental set of emotions and Russell's circumflex representation of influence. In 1971 Ekman and Freisen put in six quintessential basic emotions like disgust, joy, sadness, fear, anger and surprise which are globally presented and identified from facial expressions. The model with six basic emotion has been the most popular and usual model for recognizing emotions from their respective facial expression.

Table 1 The elements and forms of home robot face

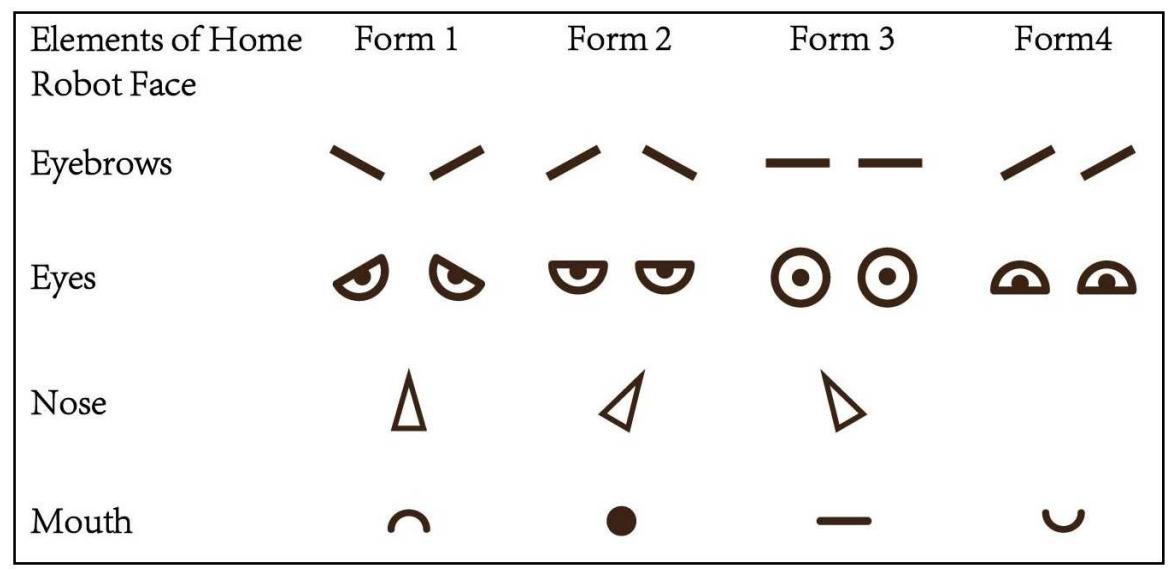

In our research, Ekman's model is utilized to analyze robots' facial expression. We also extract 4 major elements of home robot face: eyebrows, eyes, mouth and nose. There are several different 
forms for each element showed in Table 1. Facial expression of home robot is composed of different elements and forms, expect for some unreasonable styles.

\section{Kansei Measuring Method}

In our research, we collected 15 images of facial expressions shown in Fig. 1. The major elements of faces are corresponding to the forms shown in Table 1.

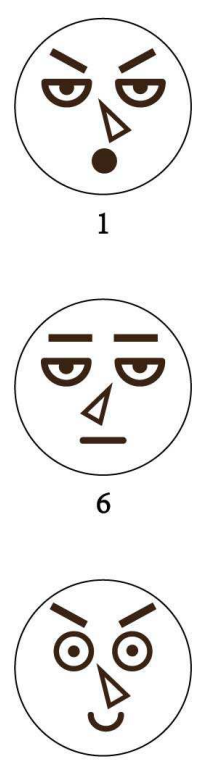

11
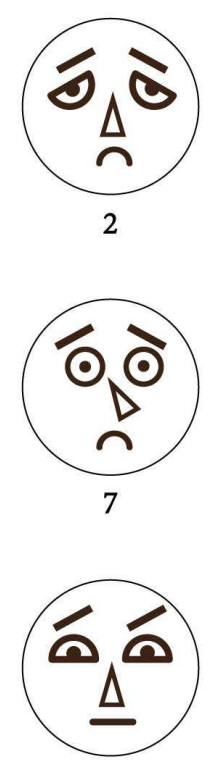

12

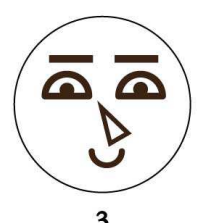

3
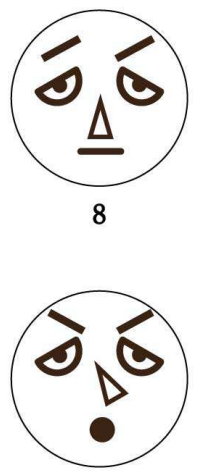

13
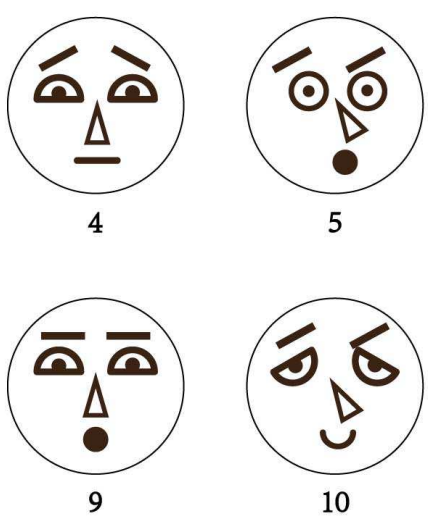

5

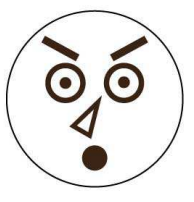

14

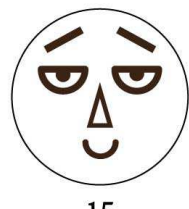

15

Figure 1. Facial Expressions of home robot in our research

Kansei words were studied to express feelings and emotions of individuals to the faces and facial expressions. When the respondents looked at a face, it could make different impressions on their feelings. The impressions reflected whether the facial expression of the image could response to one of human being's emotion. Reviewing the similar papers [6], two totally opposite words "distinct and indistinct" as Kansei words were selected to describe the feeling. We also invited 60 undergraduate students aged between 18 and 24 were asked to state their opinion and feelings on each of the selected images with respect to Likert's scale. It means that they should give 5 scores to a feeling which they may have after watched the image, whereas they should give 1 score to a feeling which they could not recognize the facial expression responding to human being's emotion clearly. If they could recognize the facial expression responding to one emotion easily, the highest score was given. The mean value of feeling scores corresponding to each image is indicated in Table 2.

\section{Statistical Analysis based on Pearson Correlation Coefficient}

Pearson Correlation Coefficient is a statistical measurement of linear Correlation between two variables. Eq. 1 gives the PCC formula of two variables $x$ and $y$ [7].

$$
r_{X y}=\frac{\sum_{i=1}^{n}\left(x_{i}-\bar{x}\right)\left(y_{i}-\bar{y}\right)}{\sqrt{\sum_{i=1}^{n}\left(x_{i}-\bar{x}\right)^{2}} \sqrt{\sum_{i=1}^{n}\left(y_{i}-\bar{y}\right)^{2}}} .
$$


Here let $x_{i}$ be the form value of each element of robot face and $y_{i}$ be the feeling score for each image. $\bar{x}$ denotes the mean value of the forms of each element, $\bar{y}$ denotes the mean value of feeling scores, and $n$ represents 30 images. $r_{x y}$ is the similarity between two variables. In particular, $r_{x y}>0$ indicates positive correlation between two variables. $r_{x y}<0$ means opposite correlation and $r_{x y}=0$ implies no correlation. Therefore, the value of $r_{X y}$ can capture the rating similarity between the two variables.

We can figure out that users' feelings for facial expression of home robot have more or less relation to the elements of robot face. It is clearly noted that the majority of facial expression is eyes and the second one is mouth in Table 3. But it is surprising result that the respondents don't have intersected with nose, which appears opposite correlation. It can conclude that designers should pay more attention to the design of eyes in order to improve user experience of home robot.

Table 3 Pearson Correlation Coefficient of elements of home robot face and facial expression

\begin{tabular}{|c|c|}
\hline Elements of Home Robot Face & Pearson Correlation Coefficient \\
\hline Eyebrows & -0.2466 \\
\hline Eyes & 0.4203 \\
\hline Nose & -0.3135 \\
\hline Mouth & 0.1571 \\
\hline
\end{tabular}

\section{Conclusion}

As design support technology, Kansei Engineering is introduced into the analysis and design of facial expression of home robot and achieves significant results in this paper. Pearson Correlation Coefficient is also applied to evaluate the relation of the elements of home robot face and facial expression. Results of the study will show that users can be attracted to eyes and eyebrows of home robots. Furthermore, the novel approach proposed in this paper will be applied in designing the products to promote user experience.

\section{References}

[1] B. Karan, A. Branko and et al: Implementation architecture of a home robot assistant, Icr2016, the Israeli Conference on Robotics (2016).

[2] W. Jitviriya and E. Hayashi: Design of emotion generation model and action selection for robots using a Self-Organizing Map, International Conference on Electrical Engineering/electronics, Computer, Telecommunications and Information Technology (2014) p.1-6.

[3] H. Inoue: Generation and kansei analysis of motions of a pet type robot based on large-small and fast-slow axes. Transactions of Japan Society of Kansei Engineering (2015).

[4] M. Li and H. B. Yan: Applying Kansei Engineering to service design: A case study of budget hotel service. International Conference on Service Systems and Service Management (2016).

[5] D. Fischinger, P. Einramhof, K. Papoutsakis and et al: Hobbit, a care robot supporting independent living at home: First prototype and lessons learned, Robotics \& Autonomous Systems (2016). Vol.75, p.60-78.

[6] T. Fong, C. Thorpe and C. Baur: Collaboration, dialogue, human-robot interaction, Robotics Research, Springer Berlin Heidelberg (2003) p.255-266.

[7] L. Sheugh and S. H. Alizadeh: A note on pearson correlation coefficient as a metric of similarity in recommender system, Ai \& Robotics (2015) p.1-6. 
[8] J. Y. Yang and D. S. Kwon: The effect of multiple robot interaction on human-robot interaction, in 2012 9th IEEE International Conference on Ubiquitous Robots and Ambient Intelligence (November, 2012) p.30-33.

[9] A. Mohd Lokman: Design \& emotion: the Kansei engineering methodology, Malaysian Journal of Computing (2010). Vol. 1, p.1-11.

[10]D. Mazzei, N. Lazzeri, D. Hanson and D. De Rossi: HEFES: An Hybrid Engine for Facial Expressions Synthesis to control human-like androids and avatars, Proc. IEEE RAS EMBS Int. Conf. Biomed. Robot. Biomechatronics (2012) p.195-200.

[11]C. Becker-Asano and H. Ishiguro: Evaluating facial displays of emotion for the android robot Geminoid F, IEEE SSCI (2011) p.22-29..

[12] T. Hashimoto, H. Kobayashi and N. Kato: Educational system with the android robot SAYA and field trial, IEEE Int. Conf. Fuzzy Syst (2011). Vol. 8, No. 3, p.766-771.

Table 2 Relationship of Kansei Words with Facial expressions in images

\begin{tabular}{|c|c|c|c|c|c|}
\hline Image ID & Eyebrows & Eyes & Nose & Mouth & Mean Value \\
\hline 1 & 1 & 2 & 3 & 2 & 2.77 \\
\hline 2 & 2 & 1 & 1 & 1 & 3.24 \\
\hline 3 & 3 & 4 & 3 & 4 & 3.04 \\
\hline 4 & 2 & 4 & 1 & 3 & 3.25 \\
\hline 5 & 4 & 3 & 3 & 2 & 2.85 \\
\hline 6 & 3 & 2 & 2 & 3 & 2.81 \\
\hline 7 & 2 & 3 & 3 & 1 & 3.53 \\
\hline 8 & 4 & 1 & 1 & 3 & 3.03 \\
\hline 9 & 3 & 4 & 1 & 2 & 3.22 \\
\hline 10 & 4 & 1 & 3 & 4 & 2.76 \\
\hline 11 & 1 & 3 & 3 & 4 & 3.81 \\
\hline 12 & 4 & 4 & 1 & 3 & 3.53 \\
\hline 13 & 1 & 1 & 3 & 2 & 2.83 \\
\hline 14 & 1 & 3 & 2 & 2 & 3.22 \\
\hline 15 & 2 & 2 & 1 & 4 & 3.56 \\
\hline
\end{tabular}

